\title{
Real-Time 3D Image Segmentation by User-Constrained Template Deformation
}

\author{
Benoît Mory, Oudom Somphone, Raphael Prevost, and Roberto Ardon \\ Medisys, Philips Research, Suresnes, France
}

\begin{abstract}
We describe an algorithm for 3D interactive image segmentation by non-rigid implicit template deformation, with two main original features. First, our formulation incorporates user input as inside/outside labeled points to drive the deformation and improve both robustness and accuracy. This yields inequality constraints, solved using an Augmented Lagrangian approach. Secondly, a fast implementation of nonrigid template-to-image registration enables interactions with a real-time visual feedback. We validated this generic technique on 21 ContrastEnhanced Ultrasound images of kidneys and obtained accurate segmentation results (Dice $>0.93$ ) in less than 3 clicks in average.
\end{abstract}

\section{Introduction}

In medical applications, segmentation of anatomical structures in difficult conditions such as tissue inhomogeneities, noise, loss-of-contrast, can be significantly facilitated by the incorporation of prior knowledge. This approach has been extensively studied in terms of shape prior by constraining the solution to remain close to a predefined shape. For instance, statistical methods have been proposed to model shapes, such as the active shape models [1]. In the level-set framework, shape priors have also been used, penalizing the dissimilarity between the implicit object representation and the one embedding the prior shape, via an additive shape constraint [2 5]. These two approaches have been combined by embedding training shapes in distance functions and defining a statistical model for the shape term [6 6 ].

Template-to-image registration is a possible alternative, recently applied to medical applications such as liver segmentation in CT [10], in which segmentation is performed by geometrically deforming a binary template towards the image 10 12]. The prior is the template itself and the shape constraint consists in a regularization of the deformation.

Shape priors may be helpful but insufficient in pathological cases with extreme variability in image features and organ shapes; expert input is then essential to guide the segmentation. Few attempts to combine shape priors and interactivity have been made in $2 \mathrm{D}[13]$. In 3D, the design of intuitive and fast interactive tools remains a key challenge. We propose a formulation of non-rigid template deformation that incorporates user constraints in the form of inside/outside labels and enables a live visual feedback of the surface evolution. 


\section{Implicit Template Deformation}

Region-based variational formulations of image segmentation consist in finding a partitioning of an image $I$ that provides the best trade-off between classification error and boundary regularity. In the case of two regions, the optimal boundary is the surface $\mathcal{S}$ solution of:

$$
\min _{\mathcal{S}}\left\{\mathcal{R}(\mathcal{S})+\int_{\text {inside } \mathcal{S}} r_{1}(\mathbf{x}) d \mathbf{x}+\int_{\text {outside } \mathcal{S}} r_{2}(\mathbf{x}) d \mathbf{x}\right\}
$$

$\mathcal{R}(\mathcal{S})$ is a regularization term, commonly chosen as the surface area. $r_{1}$ and $r_{2}$ are classification error functions in the foreground and background regions, respectively. For instance, maximum-likelihood principles suggest the choice of $\log$-likelihood terms $r_{i}(\mathbf{x})=-\log p_{i}(I(\mathbf{x}))$ for known intensity distributions $p_{1}$ and $p_{2}[14]$.

An equivalent formulation can be derived for an implicit representation of $\mathcal{S}$, with a function $\Phi: \Omega \rightarrow \mathbb{R}$, positive inside $\mathcal{S}$ and satisfying $\Phi^{-1}(0)=\mathcal{S}$. Let $H$ denote the Heaviside function ( $H(a)=1$ if $a>0,0$ otherwise); $H(\Phi)$ is the characteristic function of the region enclosed by $\mathcal{S}$ and (1) is equivalent to:

$$
\min _{\Phi}\left\{\mathcal{R}(\Phi)+\int_{\Omega} H(\Phi(\mathbf{x})) r(\mathbf{x}) d \mathbf{x}\right\} \quad \text { with } \quad r(\mathbf{x})=r_{1}(\mathbf{x})-r_{2}(\mathbf{x})
$$

Regularization $\mathcal{R}(\Phi)$ can be complemented with an additional shape prior term that enforces the solution to remain close to a predefined implicit representation. For instance, if $\Phi$ is a distance function, the shape term can penalize the $L_{2^{-}}$ distance to a globally transformed template [2]. However, this technique does not guarantee that the zero level-set of the solution preserves the topology of the prior shape. Moreover, penalizing the surface area inevitably smooths out possible important details of the prior shape.

To cope with these problems, alternative approaches have been proposed 10 12] to define $\Phi$ as the deformation of a given implicit template $\Phi_{0}$, defined in a referential $\Omega_{0}$, with a geometric transformation $\psi$ (see Fig. (1):

$$
\Phi=\Phi_{0} \circ \psi
$$

The unknown becomes the transformation $\psi$ and $\mathcal{R}(\Phi)$ in (2) is substituted with a shape term $\mathcal{R}(\psi)$, consisting in a regularization constraint acting on $\psi$.

Thus, a general formulation of image segmentation by implicit template deformation reads:

$$
\min _{\psi}\left\{E(\psi)=\mathcal{R}(\psi)+\int_{\Omega} H\left(\Phi_{0} \circ \psi(\mathbf{x})\right) r(\mathbf{x}) d \mathbf{x}\right\}
$$

Compliance with the shape prior is determined by both the deformation model $\psi$ and its associated constraint $\mathcal{R}(\psi)$. In the non-rigid case, Saddi et al. represented the deformation with a diffeomorphic fluid model [10], Somphone et al. proposed deformations based on finite elements 11], and Huang and Metaxas adopted Free Form Deformations in their Metamorphs [12]. 


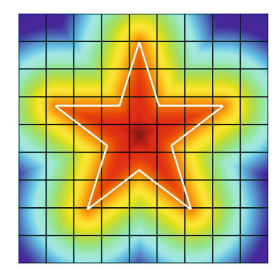

implicit template $\Phi_{0}$

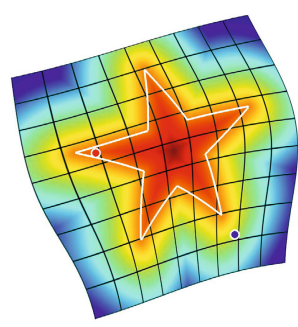

$\Phi=\Phi_{0} \circ \psi$

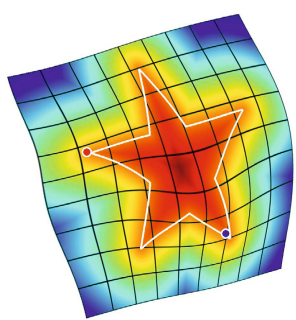

$\Phi$ satisfying constraints.

Fig. 1. Deformation of a star-shaped template (white contour) implicitly represented with a signed distance function $\Phi_{0}$, with inside (blue) and outside (red) constraints

\section{Proposed Formulation}

The aforementioned approaches of template-to-image registration do not consider possibilities for user interactions. Moreover, they are adapted from existing non-rigid image-to-image registration methods. Therefore, they inherit an algorithmic complexity that is incompatible with real-time feedback in 3D.

\subsection{User Interactions as Inequality Constraints}

Additional control can be obtained by letting the user locate specific points that lie inside/outside the object. Denoting $\left\{\mathbf{x}_{k}\right\}$ a set of $N$ labeled points, this translates into $N$ constraints on the sign of the transformed template at $\mathbf{x}_{k}$ :

$$
\forall k \in\{0, \ldots, N-1\} \quad \gamma_{k} \Phi_{0} \circ \psi\left(\mathbf{x}_{k}\right) \geq 0
$$

where $\gamma_{k}=1$ (resp. -1 ) for inside (resp. outside) points, as illustrated in Fig. 1 Note that the surface $\left\{\Phi_{0} \circ \psi=0\right\}$ can also be enforced to go through a specific point by adding both inside and outside constraints at the same location.

\subsection{Transformation Model}

In the context of template-to-image registration, the choice of a transformation model $\psi$ in (4) relates to the notion of shape. In particular, a shape should be invariant to geometric transforms such as rotation and scaling. We refer to such a global transformation as the pose. To separate pose from subsequent shape deformation, we define $\psi$ as a composition of a global transformation $\mathcal{G}$ and a local transformation $\mathcal{L}[15]$ :

$$
\psi=\mathcal{L} \circ \mathcal{G}
$$

Pose. $\mathcal{G}: \Omega \rightarrow \Omega_{0}$ is chosen as a parametric transform that globally aligns the template with the target in the image. For anatomical structures, similarities (preserving aspect ratio) are particularly adapted. Thus, $\mathcal{G}$ is defined by a matrix in homogeneous coordinates, with 7 parameters $\mathbf{p}=\left\{p_{i}\right\}_{i=1 \cdots 7}$. 
Deformation. $\mathcal{L}: \Omega_{0} \rightarrow \Omega_{0}$ is defined by a displacement field $\mathbf{u}$ in the template referential $\mathcal{L}=\mathbf{u}+\mathbf{I d}$, where $\mathbf{u}$ should be smoothly-varying in space. To take advantage of fast Gaussian filtering, the displacement $\mathbf{u}$ is defined as a smoothed version of an auxiliary displacement field $\mathbf{v}\left(K_{\sigma}\right.$ is a Gaussian of scale $\left.\sigma\right)$ :

$$
\mathbf{u}(\mathbf{x})=\left[K_{\sigma} * \mathbf{v}\right](\mathbf{x})=\int_{\Omega_{0}} K_{\sigma}(\mathbf{x}-\mathbf{y}) \mathbf{v}(\mathbf{y}) d \mathbf{y}
$$

\subsection{Shape Term}

Decomposing $\psi=\mathcal{L} \circ \mathcal{G}$ allows to define a shape term as a function of the shape deformation $\mathcal{L}$ only, regardless of the pose $\mathcal{G}$. Using the $L_{2}$ norm, we choose to constrain $\mathcal{L}$ towards the identity Id:

$$
\mathcal{R}(\mathcal{L})=\frac{\lambda}{2}\|\mathcal{L}-\mathbf{I d}\|_{2}^{2}=\frac{\lambda}{2} \int_{\Omega_{0}}\|\mathbf{u}(\mathbf{x})\|^{2} d \mathbf{x}
$$

where $\lambda$ is a positive scalar parameter. $\mathcal{R}$ quantifies the deviation of the segmentation from the prior shape by a displacement magnitude in the template referential $\Omega_{0}$. Finally, the constrained optimization problem to solve reads:

$$
\min _{\mathbf{p}, \mathbf{v}}\left\{E\left(\psi_{\mathbf{p}, \mathbf{v}}\right)=\frac{\lambda}{2} \int_{\Omega_{0}}\left\|K_{\sigma} * \mathbf{v}\right\|^{2}+\int_{\Omega} H\left(\Phi_{0} \circ \psi_{\mathbf{p}, \mathbf{v}}\right) r\right\}
$$

subject to

$$
\gamma_{k} \Phi_{0} \circ \psi_{\mathbf{p}, \mathbf{v}}\left(\mathbf{x}_{k}\right) \geq 0, \quad \forall k \in 0 . . N-1
$$

\section{Augmented Lagrangian Scheme}

To minimize the non-convex functional $E\left(\psi_{\mathbf{p}, \mathbf{v}}\right)$ under a set of $N$ non-linear inequality constraints, we follow an Augmented Lagrangian methodology [16] and define an equivalent unconstrained problem. Problem (9) is equivalent to:

$$
\begin{gathered}
\min _{\psi_{\mathbf{p}, \mathbf{v}}}\left\{\tilde{E}\left(\psi_{\mathbf{p}, \mathbf{v}}\right)=\max _{\boldsymbol{\alpha} \geq 0}\left\{E\left(\psi_{\mathbf{p}, \mathbf{v}}\right)-\sum_{k=0}^{N-1} \alpha_{k} c_{k}\left(\psi_{\mathbf{p}, \mathbf{v}}\right)\right\}\right\} \\
\text { with } \quad c_{k}\left(\psi_{\mathbf{p}, \mathbf{v}}\right)=\gamma_{k} \Phi_{0} \circ \psi_{\mathbf{p}, \mathbf{v}}\left(\mathbf{x}_{k}\right)
\end{gathered}
$$

where $\alpha_{k}$ is the Lagrange multiplier associated to the $k^{\text {th }}$ constraint. (10) has the same set of solutions as the original Problem (9): if $\psi_{\mathbf{p}, \mathbf{v}}$ satisfies all constraints $c_{k}$, then $\tilde{E}\left(\psi_{\mathbf{p}, \mathbf{v}}\right)=E\left(\psi_{\mathbf{p}, \mathbf{v}}\right)$, otherwise $\tilde{E}\left(\psi_{\mathbf{p}, \mathbf{v}}\right)=+\infty$. To avoid jumps of $\tilde{E}$ from finite to infinite values, a practical minimization requires to rely on a smooth approximation $\hat{E}$. In order to constrain the maximizers $\boldsymbol{\alpha}=\left\{\alpha_{k}\right\}$ to finite values during the iterative process, a quadratic penalty parameter $\mu$ and a set of multipliers $\boldsymbol{\alpha}^{j}$ (at the $j^{\text {th }}$ iteration) are explicitly introduced to define:

$$
\hat{E}_{\mu}\left(\psi_{\mathbf{p}, \mathbf{v}}, \boldsymbol{\alpha}^{j}\right)=\max _{\boldsymbol{\alpha} \geq 0}\left\{E\left(\psi_{\mathbf{p}, \mathbf{v}}\right)-\sum_{k=0}^{N-1} \alpha_{k} c_{k}\left(\psi_{\mathbf{p}, \mathbf{v}}\right)-\frac{1}{2 \mu} \sum_{k=0}^{N-1}\left(\alpha_{k}-\alpha_{k}^{j}\right)^{2}\right\}
$$


In (11), optimal Lagrange multipliers associated to each constraint $c_{k}\left(\psi_{\mathbf{p}, \mathbf{v}}\right)$ can be found as a function of previously estimated values:

$$
\alpha_{k}^{j+1}= \begin{cases}0 & \text { if } \alpha_{k}^{j}-\mu c_{k}\left(\psi_{\mathbf{p}, \mathbf{v}}\right) \leq 0 \\ \alpha_{k}^{j}-\mu c_{k}\left(\psi_{\mathbf{p}, \mathbf{v}}\right) & \text { otherwise }\end{cases}
$$

Substituting (12) in (11) yields the expression of the smooth approximation $\hat{E}_{\mu}$ :

$$
\begin{gathered}
\hat{E}_{\mu}\left(\psi_{\mathbf{p}, \mathbf{v}}, \alpha^{j}\right)=E\left(\psi_{\mathbf{p}, \mathbf{v}}\right)+\sum_{k=0}^{N-1} \Psi_{\mu}\left(c_{k}\left(\psi_{\mathbf{p}, \mathbf{v}}\right), \alpha_{k}^{j}\right) \\
\text { with } \Psi_{\mu}(a, b)= \begin{cases}-a b+\frac{\mu}{2} a^{2} & \text { if } \mu a \leq b \\
-\frac{1}{2 \mu} b^{2} & \text { otherwise. }\end{cases}
\end{gathered}
$$

Finally, the alternate scheme below provides at convergence a local minimizer of (9) that satisfies all inequality constraints.

given starting penalty parameter $\mu^{0}$, and $\alpha^{0}=0$,

repeat

choose $\mu^{t}>\mu^{t-1}$

repeat

(A) $\psi_{\mathbf{p}, \mathbf{v}}$ fixed, update $\boldsymbol{\alpha}^{j+1}$ as in (12)

(B) $\boldsymbol{\alpha}^{j}$ fixed, update $\psi_{\mathbf{p}, \mathbf{v}}$ by minimizing (13)

until convergence;

until a local minimum of $E\left(\psi_{\mathbf{p}, \mathbf{v}}\right)$ satisfying $\forall k, c_{k}\left(\psi_{\mathbf{p}, \mathbf{v}}\right) \geq 0$ is found;

In our application, the effect of each interaction is visualized with a real-time display of the surface evolution. This relies on fast iterations of the minimization of (13) involved in step (B), jointly performed with respect to $\mathbf{p}$ and $\mathbf{v}$ by gradient descent of:

$$
\hat{E}(\mathbf{p}, \mathbf{v})=E\left(\psi_{\mathbf{p}, \mathbf{v}}\right)+\sum_{k=0}^{N-1} \Psi_{\mu}\left(c_{k}(\mathbf{p}, \mathbf{v}), \alpha_{k}^{j}\right)
$$

Evolution equations for each pose parameter $p_{i}$ and the displacement field $\mathbf{v}$ are:

$$
\begin{array}{rr}
\frac{\partial p_{i}}{\partial t}=-\frac{\partial \hat{E}}{\partial p_{i}}= & -\int_{\Omega_{0}} \delta\left(\Phi_{0} \circ \mathcal{L}\right) r \circ \mathcal{G}^{-1} \mathcal{A}_{i}-\sum_{k=0}^{N-1} b_{k} \mathcal{A}_{i} \circ \mathcal{G}\left(\mathbf{x}_{k}\right) \\
\frac{\partial \mathbf{v}}{\partial t}=-\frac{\partial \hat{E}}{\partial \mathbf{v}}=-K_{\sigma} *\left[\lambda \mathbf{u}+\frac{\left(\delta\left(\Phi_{0} \circ \mathcal{L}\right) r \circ \mathcal{G}^{-1}\right.}{\text { shape }}+\frac{\left.\sum_{k=0}^{N-1} b_{k} \delta_{\mathcal{G}\left(\mathbf{x}_{k}\right)}\right)}{\text { image force }} \nabla \Phi_{0} \circ \mathcal{L}\right]
\end{array}
$$


where $b_{k}=\gamma_{k} \frac{\partial \Psi_{\mu}}{\partial a}, \mathcal{A}_{i}(\mathbf{x})=\left\langle\nabla \Phi_{0} \circ \mathcal{L}(\mathbf{x}),\left(\mathbf{I}+\mathbf{J}_{\mathbf{u}(\mathbf{x})}\right) \frac{\partial \mathcal{G}}{\partial p_{i}} \circ \mathcal{G}^{-1}(\mathbf{x})\right\rangle$ with $\mathbf{I}$ the Identity matrix and $\mathbf{J}_{\mathbf{u}}$ the Jacobian matrix of $\mathbf{u}$, and $\delta_{\mathcal{G}\left(\mathbf{x}_{k}\right)}(\mathbf{x})=\delta\left(\mathbf{x}-\mathcal{G}\left(\mathbf{x}_{k}\right)\right)$.

Let us now emphasize key properties of (16) that enable a fast implementation. First, interpolating $\Phi_{0} \circ \mathcal{L}$ and $\nabla \Phi_{0} \circ \mathcal{L}$ over the whole domain $\Omega_{0}$ would be extremely time-consuming. Nevertheless, since it is multiplied either by $\delta\left(\Phi_{0} \circ \mathcal{L}\right)$ or $\delta_{\mathcal{G}\left(\mathbf{x}_{k}\right)}$, the warped gradient $\nabla \Phi_{0} \circ \mathcal{L}$ is only needed on the set $\left\{\Phi_{0} \circ \mathcal{L}=0\right\}$ and at points $\left\{\mathbf{x}_{k}\right\}$ (Fig. 2, a) which highly reduces warped gradient computations.

Moreover, precise knowledge of the warped template $\Phi_{0} \circ \mathcal{L}$ is only necessary near its zero level. Setting $\Phi_{0}$ to a distance function to the prior shape allows a coarse-to-fine approach using octrees. At each level, decision is made to further refine each cell based on the distance value (Fig. 2,b) which also significantly reduces the warping complexity.

Finally, a benefit of the displacement model (7) is that image forces and constraints extrapolate to the whole space with a convolution with $K_{\sigma}$ (Fig. 2. c).

Our 3D implementation supports about 100 time steps per second when discretizing $\Omega_{0}$ on a lattice of $48^{3}$ points, which allows a live response to constraints.

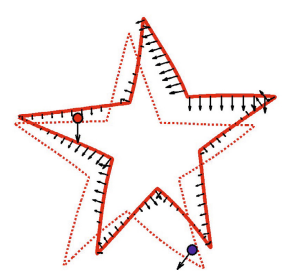

(a) surface/point forces

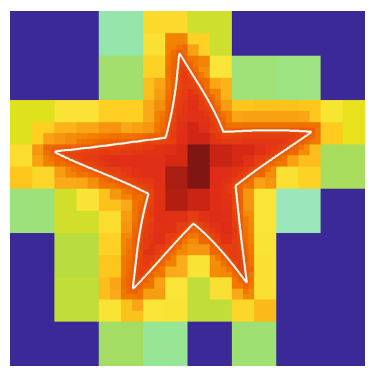

(b) hierarchical warp $\phi_{0} \circ \mathcal{L}$

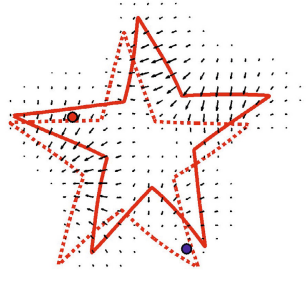

(c) convolved deformation

Fig. 2. Fast deformation of a distance function with hierarchical warp and convolution

\section{Segmenting Kidneys in Contrast-Enhanced Ultrasound}

We validated this method on $3 \mathrm{D}$ contrast-enhanced ultrasound (CEUS) images of kidneys. CEUS is a recent imaging modality that allows to visualize blood flow in real-time without any risk for the patient. However, segmenting kidneys in CEUS images is difficult : contrast agents generate noisy data, limited field of view of probes often prevents the acquisition of the whole kidney and lesions induce variations from the usual shape. Unlike in conventional ultrasound, very few methods have been reported for 3D CEUS segmentation.

Validation was performed on a representative dataset of 21 CEUS volumes acquired on a Philips iU22 ultrasound system with different probes (V6-2 and X6-1), resolutions and fields of view. Typical size of the images is $512 \times 320 \times 256$. For each case, ground truth segmentation was provided by a radiologist. 
Template $\Phi_{0}$ is set to an ellipsoid. Segmentation criterion is the image gradient flux across the boundary, which is equivalent to a region-based formulation such as (4) with $r(\mathbf{x})=\Delta I(\mathbf{x})$ where $\Delta$ is the Laplacian operator. The scale $\sigma$ of the deformation field in (7) is set to $25 \mathrm{~mm}$.
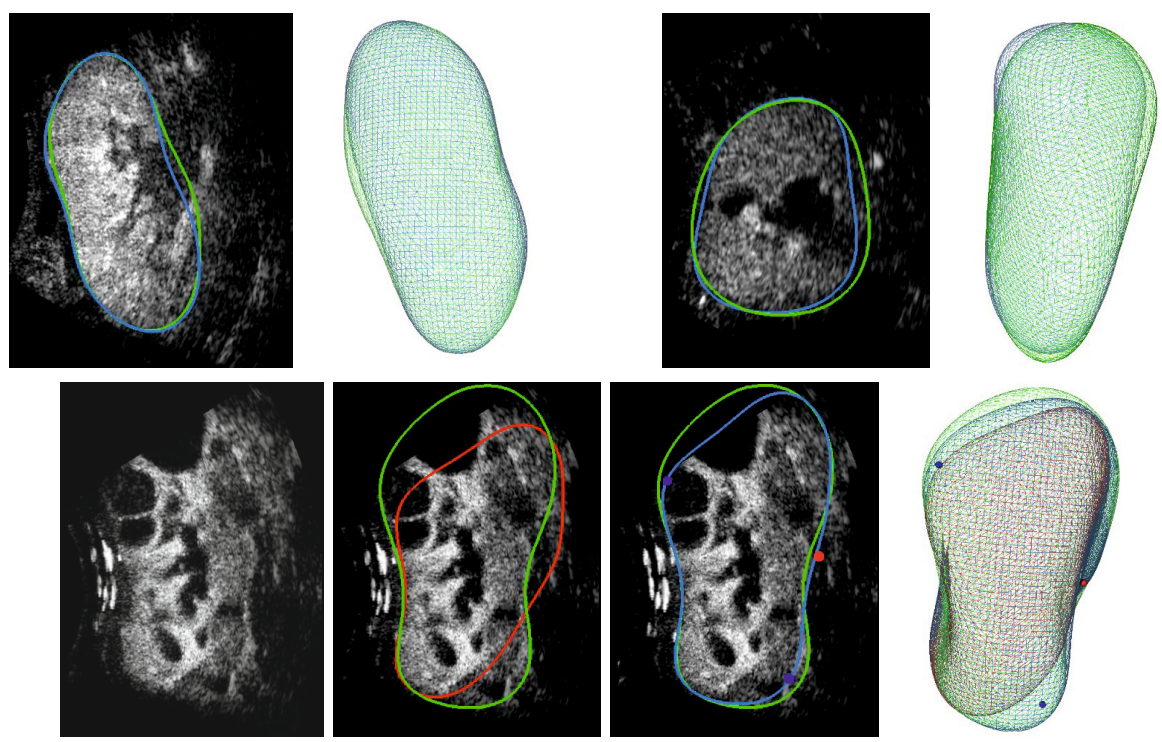

(a)

(b)

(c)

(d)

Fig. 3. Top: automatic segmentation (blue) and ground truth (green) for 2 patients. Bottom: segmentation with user interactions. (a) slice of the CEUS volume. (b) ground truth (green) and automatic segmentation (red). (c) corrected segmentation (blue) with 3 clicks. (d) ground truth (green), automatic (red) and after corrections (blue).

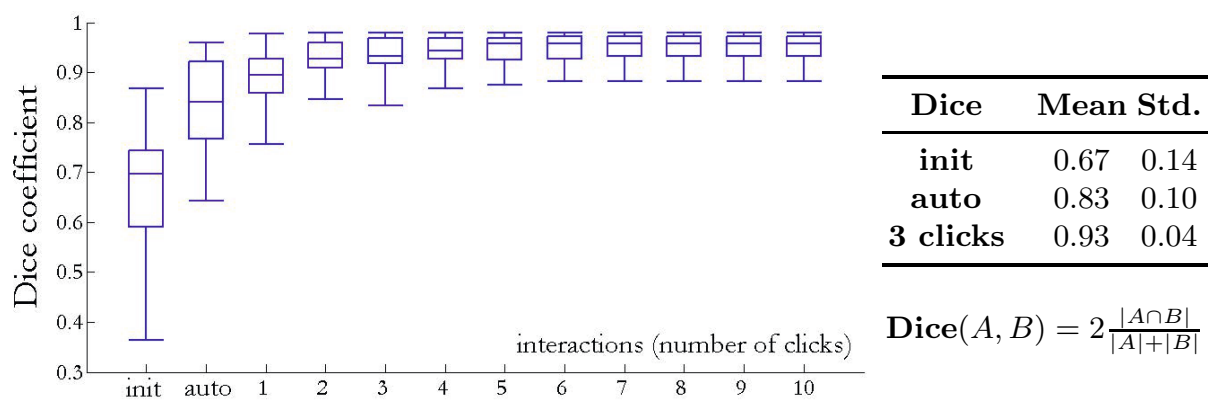

Fig. 4. Comparison with ground truth, as a function of the number of interactions

While automatic segmentation is successful in some cases, difficult cases with cysts and partial visibility of the kidney (Fig. 3) inevitably require corrections. Fig. 4 summarizes the results obtained by a trained user and quantifies how 
segmentation performance improves with the number of interactions. In most cases, three clicks are sufficient to obtain a satisfactory result (Dice $0.93 \pm 0.04$ ).

\section{Conclusion}

Although proven a solid approach for medical image segmentation, template deformation is still unable to cope with the variety of shapes that pathologies generate. Reliable interactions are essential add-ons to these segmentation tools. In this context, we introduced user corrections in a template deformation framework with simple clicks inside/outside the object, with special care devoted to algorithmic efficiency to enable real-time $3 \mathrm{D}$ visualization and intuitive control.

\section{References}

1. Cootes, T.F., et al.: Active shape models: Their training and application. CVIU 61(1), 38-59 (1995)

2. Paragios, N., Rousson, M., Ramesh, V.: Matching Distance Functions: A Shapeto-Area Variational Approach for Global-to-Local Registration. In: Heyden, A., Sparr, G., Nielsen, M., Johansen, P. (eds.) ECCV 2002, Part II. LNCS, vol. 2351, pp. 775-789. Springer, Heidelberg (2002)

3. Cremers, D., et al.: Towards recognition-based variational segmentation using shape priors and dynamic labeling. In: Scale Space, pp. 388-400 (2003)

4. Chan, T.F., Zhu, W.: Level set based shape prior segmentation. In: IEEE CVPR, pp. II:1164-II:1170 (2005)

5. Foulonneau, A., et al.: Multi-reference shape priors for active contours. IJCV 81(1), 68-81 (2009)

6. Leventon, M.E., et al.: Statistical shape influence in geodesic active contours. In: IEEE CVPR, pp. 316-323 (2000)

7. Tsai, A., et al.: A shape-based approach to the segmentation of medical imagery using level sets. IEEE TMI 22(2), 137-154 (2003)

8. Bresson, X., et al.: A variational model for object segmentation using boundary information and shape prior driven by the Mumford-Shah functional. IJCV 68(2), 145-162 (2006)

9. Cremers, D., et al.: Shape statistics in kernel space for variational image segmentation. Pattern Recognition 36(9), 1929-1943 (2003)

10. Saddi, K.A., et al.: Global-to-local shape matching for liver segmentation in ct imaging. In: MICCAI (October 2007)

11. Somphone, O., Mory, B., Makram-Ebeid, S., Cohen, L.: Prior-Based PiecewiseSmooth Segmentation by Template Competitive Deformation Using Partitions of Unity. In: Forsyth, D., Torr, P., Zisserman, A. (eds.) ECCV 2008, Part III. LNCS, vol. 5304, pp. 628-641. Springer, Heidelberg (2008)

12. Huang, X., Metaxas, D.: Metamorphs: Deformable shape and appearance models. IEEE Trans. PAMI 30(8), 1444-1459 (2008)

13. Freedman, D., Zhang, T.: Interactive graph cut based segmentation with shape priors. In: CVPR, vol. 1, pp. 755-762 (June 2005)

14. Zhu, S.C., Yuille, A.: Region competition: Unifying snakes, region growing, and bayes/mdl for multiband image segmentation. PAMI 18(9), 884-900 (1996)

15. Yezzi, A., Soatto, S.: Deformotion: Deforming motion, shape average and the joint registration and approximation of structures in images. IJCV 53(2), 153-167 (2003)

16. Nocedal, J., Wright, S.J.: Numerical Optimization. Springer (August 1999) 